\title{
Image Quality Analysis of Various Gastrointestinal Endoscopes: Why Image Quality Is a Prerequisite for Proper Diagnostic and Therapeutic Endoscopy
}

\author{
Weon Jin Ko ${ }^{1}$, Pyeong An ${ }^{2}$, Kwang Hyun Ko ${ }^{1}$, Ki Baik Hahm¹, Sung Pyo Hong ${ }^{1}$ and Joo Young Cho ${ }^{1}$ \\ ${ }^{1}$ Digestive Disease Center, CHA Bundang Medical Center, CHA University, Seongnam, ${ }^{2}$ Digestive Disease Center, Soonchunhyang \\ University Hospital, Soonchunhyang University College of Medicine, Seoul, Korea
}

\begin{abstract}
Arising from human curiosity in terms of the desire to look within the human body, endoscopy has undergone significant advances in modern medicine. Direct visualization of the gastrointestinal (GI) tract by traditional endoscopy was first introduced over 50 years ago, after which fairly rapid advancement from rigid esophagogastric scopes to flexible scopes and high definition videoscopes has occurred. In an effort towards early detection of precancerous lesions in the GI tract, several high-technology imaging scopes have been developed, including narrow band imaging, autofocus imaging, magnified endoscopy, and confocal microendoscopy. However, these modern developments have resulted in fundamental imaging technology being skewed towards red-green-blue and this technology has obscured the advantages of other endoscope techniques. In this review article, we have described the importance of image quality analysis using a survey to consider the diversity of endoscope system selection in order to better achieve diagnostic and therapeutic goals. The ultimate aims can be achieved through the adoption of modern endoscopy systems that obtain high image quality.
\end{abstract}

Clin Endosc 2015;48:374-379

Key Words: Image quality; Videoscope; Technology; Advancement

\section{INTRODUCTION}

Gastrointestinal (GI) endoscopy is the most fundamental and prerequisite clinical examination in order to diagnose and treat various diseases that occur in the GI tract. After electronic endoscopes and fiber optic endoscopy were developed in 1983, the endoscopic sector advanced rapidly, resulting in video endoscopes, with built-in coupled charged device (CCD) being developed and subsequently introduced into the clinic in 1987. This completed the development of the endoscopic system most commonly used by gastroenterologists today. With

Received: February 14, 2015 Revised: June 30, 2015

Accepted: August 4, 2015

Correspondence: Joo Young Cho

Digestive Disease Center, CHA Bundang Medical Center, CHA University, 59 Yatap-ro, Bundang-gu, Seongnam 13496, Korea

Tel: +82-31-780-5005, Fax: +82-31-780-5219, E-mail: cjy6695@chamc.co.kr

(c) This is an Open Access article distributed under the terms of the Creative Commons Attribution Non-Commercial License (http://creativecommons.org/ licenses/by-nc/3.0) which permits unrestricted non-commercial use, distribution, and reproduction in any medium, provided the original work is properly cited. further advancement of imaging technology, the system was recently converted to high definition images with enhanced resolution. ${ }^{2}$ Since image quality is the most important factor in observing suspicious lesions with a gastroscope or colonoscope, we have performed a survey to analyse diverse globally available endoscope systems from Olympus (Tokyo, Japan), Pentax (Tokyo, Japan), Fujinon (Fujifilm, Tokyo, Japan), and, recently, Karl Storz (Tuttlingen, Germany), with a focus on comparing image quality. Since a survey comparing image quality between products and determine the differences has not, to date, been performed, a prospective study to compare the image quality from various endoscopes might be prerequisite.

\section{RED-GREEN-BLUE AND REAL IMAGE TECHNOLOGY FOR ENDOSCOPE IMAGING: THE EVOLUTION OF ENHANCED IMAGING SYSTEMS AFTER IMAGING INNOVATIONS}

In video endoscopes, the $\mathrm{CCD}$ is mounted on the tip of the 
endoscope and serves as the image-sensing device, in which color separation is achieved through use of a red-green-blue (RGB) filter housed within the light source unit. The RGB filter consists of three band filters and covers all wavelengths of the visible spectrum, ranging from approximately 400 to $800 \mathrm{~nm}^{3}$ In this RGB-based endoscopy system, narrow band imaging (NBI) is an endoscopic technique that may enhance the accuracy of diagnosis using narrow-bandwidth filters in an RGB system. ${ }^{4}$ The basic principle of NBI is that the depth of penetration of light into the mucosa depends on the wavelength of that light; deep penetration is achieved with red light, intermediate penetration with green light and only superficial penetration with blue light. ${ }^{5}$ Because GI cancers originate in the mucosa, the use of blue, light of a short-wavelength, which can penetrate only into the mucosa, may be useful for early detection of GI cancer. Since black and white images have a low resolution, the light absorbed by the CCD is composited into RGB to generate color images. ${ }^{6}$ I-scan technology is the newly-developed image-enhanced endoscopic technology from Pentax. It consists of three types of algorithms: surface enhancement (SE), contrast enhancement (CE), and tone enhancement (TE). SE improves light/dark contrast and allows for detailed observation of the mucosal surface structure. CE adds blue color in relatively dark areas and allows one to distinguish subtle irregularities around the mucosal surface. With TE, the RGB components of an ordinary endoscopic image are disintegrated into each component, followed by a re-synthesis to yield a reconstructed image. In the Olympus system, NBI is an optical-filter technology that uses two narrow-band filters to provide tissue illumination in the blue and green light spectra. ${ }^{7}$ Color management technology has progressed a great deal, yet there remains a limitation in
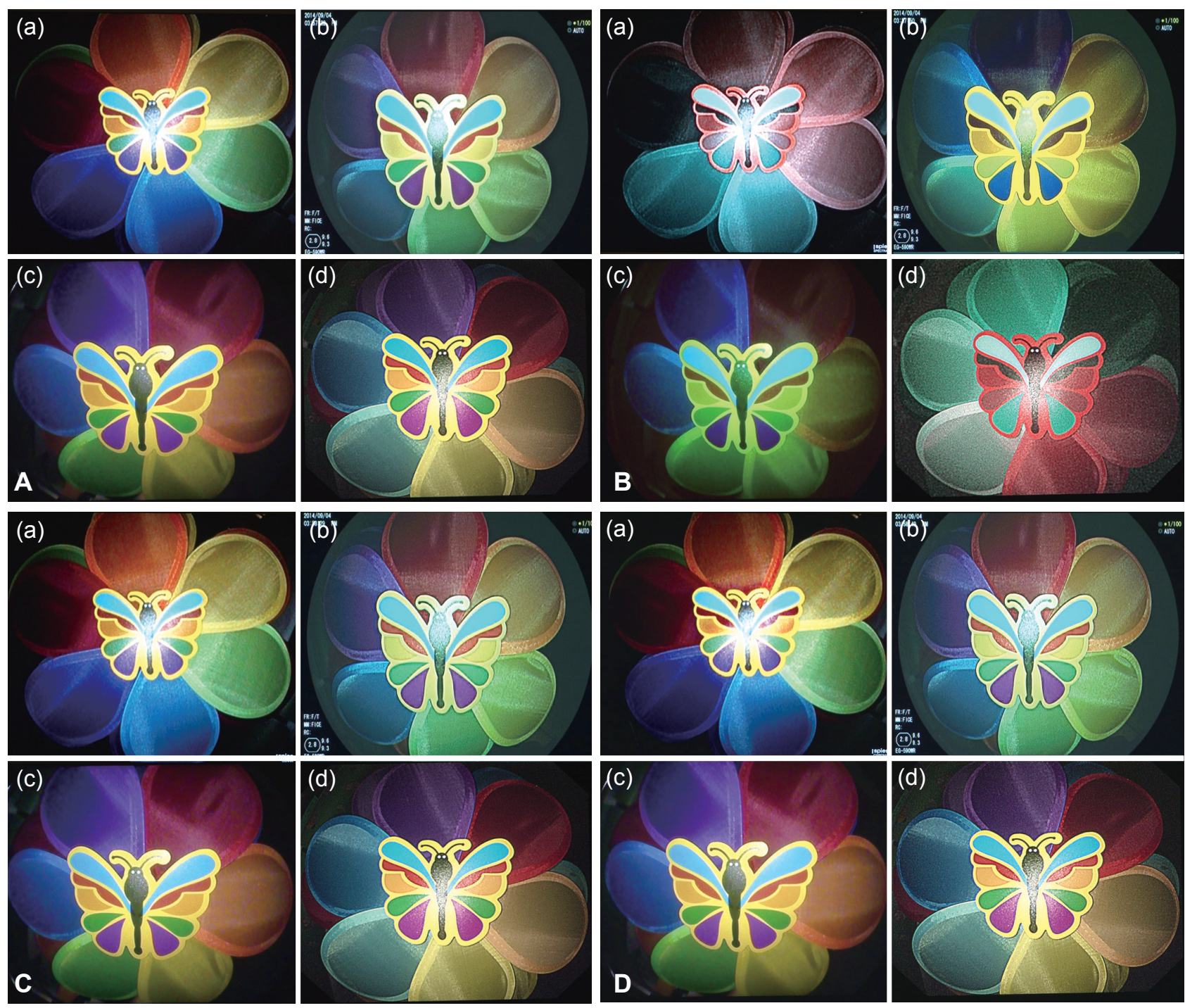

Fig. 1. Butterfly pattern (in vitro) taken according to each endoscopy company, analyzed on each point of evaluation, namely (A) definition, (B) boundary, (C) brightness, and (D) light and shade. 
terms of color reproducibility when employing RGB three primary color systems. In addition, the RGB values obtained in conventional systems often have different meanings, depending on the device characteristics or color processing. For example, many conventional color imaging systems are designed for user preference; thus, the RGB values do not represent objective color information. As a result, the RGB signal does not display one-to-one correspondence to the tri-stimulus values perceived by human vision. ${ }^{8}$ Optimal band imaging (OBI), the generic term for flexible spectral imaging color enhancement, enhances the visualization of mucosal structure and microcirculation by the selection of spectral transmittance of a dedicated wavelength. In contrast to RGB, in which the bandwidth of the spectral transmittance is narrowed by optical filters, the OBI system is based on a new computed spectral-estimation technique. In OBI, the endoscopist can select 60 spectral images per $5 \mathrm{~nm}$ at visible wavelengths between 400 and $695 \mathrm{~nm} .{ }^{9,10}$ IMAGE 1 Storz Professional Image Enhancement Software (SPIES) is the newly developed color spectrum shifting technology from Karl Storz. SPIES SPECTRA allows for the recognition of the finest tissue structures. The bright red portions of the visible spectrum are filtered out and the remaining color portions are expanded, making it easier to differentiate between tissue types. The light/dark contrast is enhanced by obtaining luminance intensity data for each pixel and applying an algorithm that allows for the detailed observation of mucosal surface structures. SPIES CLARA, on the other hand, supports proper illumination in each part of the endoscopic image, allowing for a clear display of details in both the light and dark areas of the image. Finally, SPIES CHROMA intensifies the color contrast in the image; thus, clearly visible structure surfaces are given added empha-
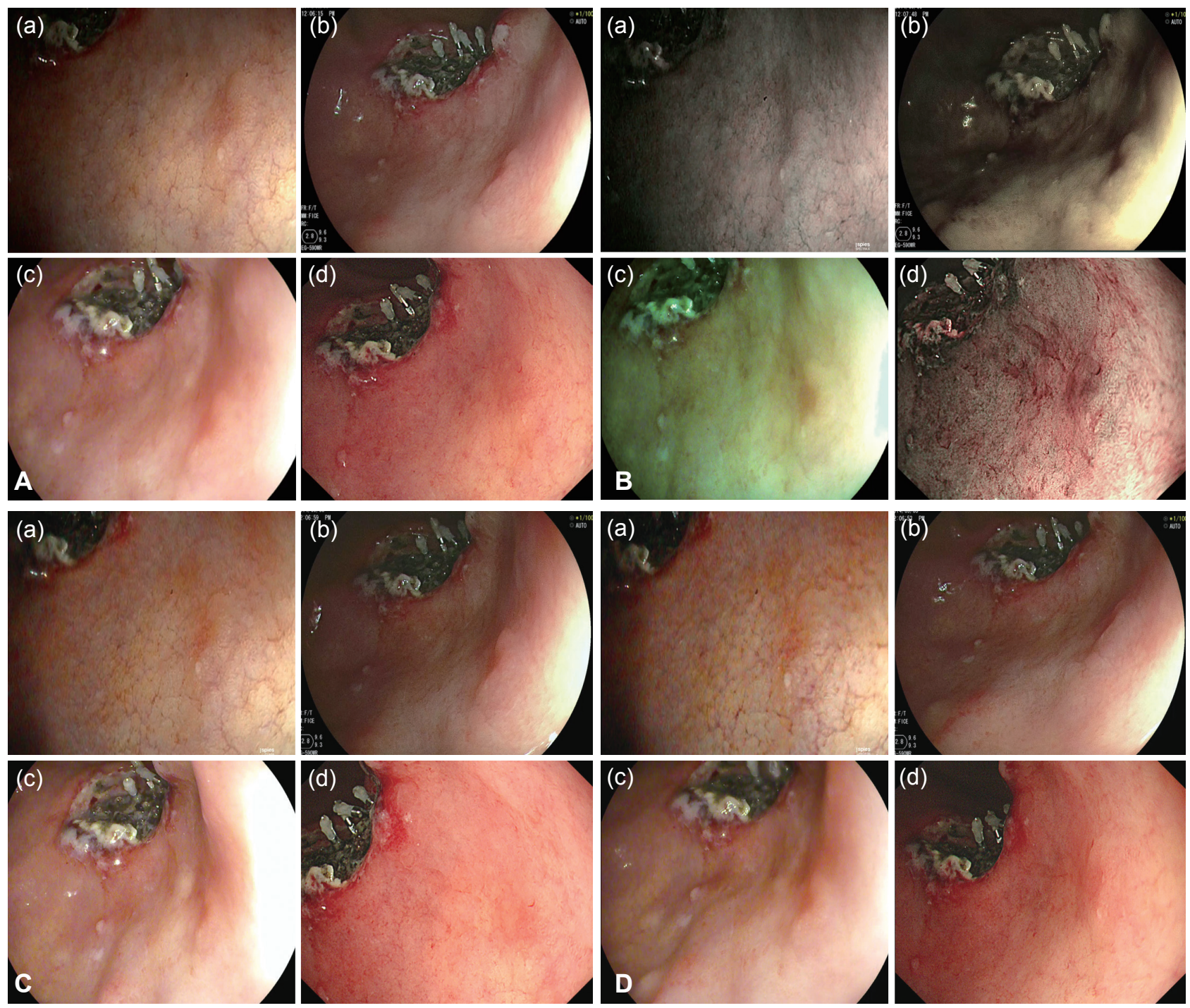

Fig. 2. Gastric lesion (in vivo) taken according to each endoscopy company analyzed on each point of evaluation, namely (A) definition, (B) boundary, (C) brightness, and (D) light and shade. 
sis while retaining the natural color perception of the image.

\section{SURVEY TO COMPARE THE IMAGE QUALITY ANALYSIS ACCORDING TO VARIOUS ENDOSCOPE SYSTEMS}

Preferences for image quality of products from the four leading companies were surveyed at 4th September 2014. For this survey, Karl Storz (model, Image 1 SPIES; scope, Silver scope $13825 \mathrm{NKS}$ ), Fujinon (model, EPX-4450HD; scope, 590WR), Olympus (model, CLV-290SL; scope, GIF-H290), and Pentax (model, EPK i7000; scope, EEG29-i10) endoscopes, with the highest image resolution among the products from each of the four companies on the market today, were selected and compared objectively. Preferences were divided into four elements that determine image quality, including elements of definition, boundaries, brightness, and 'light and shade.' A video indicating the butterfly pattern (in vitro) (Fig. 1) and actual early gastric cancer $\Pi$ a lesions (in vivo) (Fig. 2) on the endoscopic screen was shown to study participants and they were required to respond to the survey. In particular, unique spectral images from each endoscope were compared for boundaries. Comparisons were also performed with images generated using functions unique to the Karl Storz system, since its products were equipped with unique functions for brightness and 'light and shade.' The 209 respondents that participated in the current survey for image quality analysis were made up of 26 nurses, 32 medical students, and 151 GI endoscopy specialists. Based on the outcome of the survey, the selection rate of each element was calculated and analysis was conducted to determine whether there was agreement in the selections for definition, boundaries, brightness, and 'light and shade. The agreement, in terms of these selections, between the butterfly pattern and gastric lesions was also analyzed for each of the four elements (kappa value, $\mathrm{k}$ value). All statistical analyses were performed using SPSS version 19 (IBM Co., Armonk, NY, USA). In terms of the results of the survey, a total of 209 people participated in the survey and there were no missing values. For the purposes of the survey, (a), (b), (c), and (d) shall represent Karl Storz, Fujinon, Pentax, and Olympus, respectively. In the survey results for the butterfly pattern, the selection rate in terms of definition was in the order of $(c, 56.9 \%)>(d, 36.8 \%)>(a, 5.3 \%)>(b$, $1 \%)$; in terms of boundaries was $(\mathrm{d}, 50.2 \%)>(\mathrm{c}, 32.5 \%)>(\mathrm{a}$, $8.6 \%)=(\mathrm{b}, 8.6 \%)$; brightness was $(\mathrm{a}, 32.1 \%)>(\mathrm{b}, 30.6 \%)>(\mathrm{c}$, $24.9 \%)>(\mathrm{d}, 12.4 \%)$; 'light and shade' was $(\mathrm{a}, 36.4 \%)>(\mathrm{c}$, $34 \%)>(\mathrm{d}, 27.3 \%)>(\mathrm{b}, 2.4 \%)$; and the overall preferences were in order of $(c, 37.1 \%)>(d, 31.7 \%)>(a, 20.6 \%)>(b, 10.6 \%)$. In the survey results for the gastric lesions, selection rate for definition was in the order of $(\mathrm{a}, 34.9 \%)>(\mathrm{b}, 33.5 \%)>(\mathrm{d}, 23 \%)>(\mathrm{c}$, $8.6 \%)$; boundaries were $(\mathrm{d}, 48.3 \%)>(\mathrm{b}, 32.1 \%)>(\mathrm{a}, 11.5 \%)>(\mathrm{c}$, $8.1 \%)$; brightness was $(\mathrm{c}, 44 \%)>(\mathrm{d}, 24.9 \%)>(\mathrm{b}, 19.1 \%)>(\mathrm{a}, 12 \%)$; 'light and shade' was $(\mathrm{a}, 32.1 \%)>(\mathrm{b}, 29.7 \%)>(\mathrm{d}, 26.3 \%)>(\mathrm{c}$, $12 \%)$; and the overall preferences were in the order of (d,

Table 1. Preferences for the Images of the Butterfly Pattern

\begin{tabular}{lccccc}
\hline Company & Definition & Boundary & Brightness & Light and shade & Total \\
\hline Karl Storz (a) & $11(5.3)$ & $18(8.6)$ & $67(32.1)$ & $76(36.4)$ & $172(20.6)$ \\
Fujinon (b) & $2(1)$ & $18(8.6)$ & $64(30.6)$ & $5(2.4)$ & $89(10.6)$ \\
Pentax (c) & $119(56.9)$ & $68(32.5)$ & $52(24.9)$ & $71(34)$ & $310(37.1)$ \\
Olympus (d) & $77(36.8)$ & $105(50.2)$ & $26(12.4)$ & $57(27.3)$ & $265(31.7)$ \\
\hline
\end{tabular}

Values are presented as number (\%).
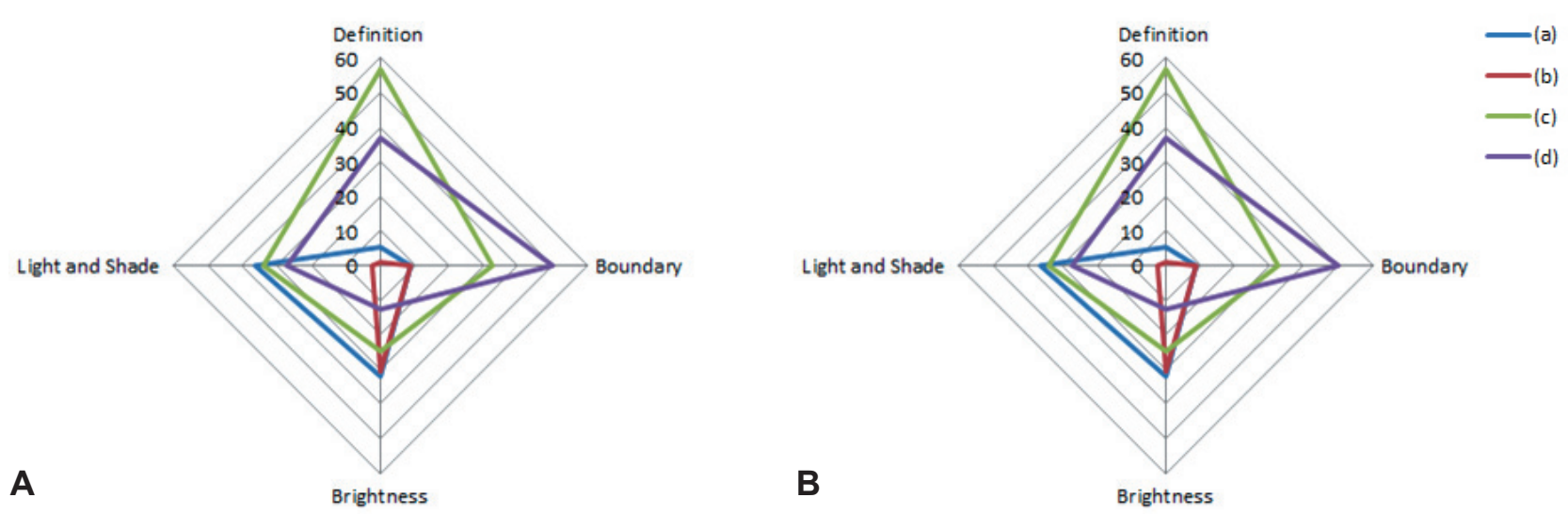

Fig. 3. Comparison of preference per product. (A) Butterfly pattern. (B) Gastric lesion. 
Table 2. Preferences for Gastric Lesion

\begin{tabular}{lccccc}
\hline Company & Definition & Boundary & Brightness & Light and shade & Total \\
\hline Karl Storz (a) & $73(34.9)$ & $24(11.5)$ & $25(12)$ & $67(32.1)$ & $189(22.6)$ \\
Fujinon (b) & $70(33.5)$ & $67(32.1)$ & $40(19.1)$ & $62(29.7)$ & $239(28.6)$ \\
Pentax (c) & $18(8.6)$ & $17(8.1)$ & $92(44)$ & $25(12)$ & $152(18.1)$ \\
Olympus (d) & $48(23)$ & $101(48.3)$ & $52(24.9)$ & $55(26.3)$ & $256(30.6)$ \\
\hline
\end{tabular}

Values are presented as number (\%).

$30.625 \%)>(\mathrm{b}, 28.6 \%)>(\mathrm{a}, 22.625 \%)>(\mathrm{c}, 18.175 \%)$ (Tables 1,2 , Fig. 3). A k value was calculated to determine whether there was agreement in the selection of definition, boundaries, brightness and 'light and shade.' However, the agreement in terms of selection between each product family was low, with a maximum $\mathrm{k}$ value of 0.117 . The agreement in selection for definition, boundaries, brightness, and 'light and shade' between the butterfly pattern and gastric lesions was also low.

\section{WHY DIVERSE ENDOSCOPIC SYSTEMS SHOULD BE A PREREQUISITE IN THE CLINIC}

An endoscope is a medical instrument used to examine the interior of a body cavity or organ by insertion of the instrument into the body. Disease in the body can be diagnosed and direct biopsy sampling can be feasibly performed under vision with an endoscope. Aside from diagnosis, therapeutic endoscopy can also be performed in some cases. The basic structure of an endoscope consists largely of a probe that is inserted into the body to generate image information, a controller that operates the probe, and a medical image visualization system that visualizes the image information. ${ }^{11}$ For instance, early neoplasia lesions usually occur at the surface of mucous membranes and a common occurrence is microvascular proliferation at the mucosal surface layer. ${ }^{12}$ Since early cancer is literally a very early state of the disease, distinguishing it from the surrounding normal tissue by visual identification under a normal endoscope is challenging. ${ }^{13}$ However, endoscopes with specialized functions, such as magnification endoscopes, NBI endoscopes, and other spectroscopy can yield higher detection and delineation in order to facilitate therapeutic intervention in addition to high detection. These advancements in optical technologies have enabled an increase in the detection rate for even precancerous lesions before overt malignancy. ${ }^{14}$ The objective of our current survey was to identify differences in the image quality of products from the leading companies in the world's endoscope market today, without selection bias or conflict of interest.

\section{PERSPECTIVES TO HIGHLIGHT IMAGE QUALITY}

Unfamiliarity and unease in adapting to a new instrument can lead to a preference for the products of a certain endoscopy company, manifesting as a monopoly on such products by one particular company. However, such situations are not helpful to either doctors or patients. Endoscope selection requires a broad range of choices of instrument, since each product has its own advantages and disadvantages depending on the training required and frequency of use. The development of instruments and competitive pricing of products can be expected with a wide range of choices, which prevents the monopolistic position of particular products. As far as diagnosis and treatment is concerned, image quality is the most important factor, for which the introduction of diverse endoscope technologies should be considered. Not only is the lens used to capture the image significant, but the technologies used to correct and process the obtained image are also important.

\section{CONCLUSIONS}

We look forward to the use of endoscopes developed in our own country with are of a higher quality, but developed under the smart and qualified imaging technologies, for which gastroenterologists should be trained with diverse, technology-based endoscopy beyond the most popularly adapted RGB technology.

Conflicts of Interest

The authors have no financial conflicts of interest.

\section{REFERENCES}

1. Classen M, Knyrim K, Seidlitz HK, Hagenmüller F. Electronic endoscopy: the latest technology. Endoscopy 1987;19:118-123.

2. Bruno MJ. Magnification endoscopy, high resolution endoscopy, and chromoscopy: towards a better optical diagnosis. Gut 2003;52 Suppl 4:iv7-iv11.

3. Muto M, Horimatsu T, Ezoe Y, Morita S, Miyamoto S. Improving visualization techniques by narrow band imaging and magnification endoscopy. J Gastroenterol Hepatol 2009;24:1333-1346. 
4. Tajiri H, Matsuda K, Fujisaki J. What can we see with the endoscope? Present status and future perspectives. Dig Endosc 2002;14:131-137.

5. Sano Y, Muto M, Tajiri H, Ohtsu A, Yoshida S. Optical/digital chromoendoscopy during colonoscopy using narrow-band imaging system Dig Endosc 2005;17(Suppl 1):S43-S48.

6. Cho WY, Jang JY, Lee DH; Endoscopic Technology and Investigation Study Group. Recent advances in image-enhanced endoscopy. Clin Endosc 2011;44:65-75.

7. Kodashima S, Fujishiro M. Novel image-enhanced endoscopy with i-scan technology. World J Gastroenterol 2010;16:1043-1049.

8. Yamaguchi M, Haneishi H, Ohyama N. Beyond red-green-blue (rgb): spectrum-based color imaging technology. J Imaging Sci Technol 2008;52:10201-1-10201-15.

9. Osawa $H$, Yoshizawa M, Yamamoto $H$, et al. Optimal band imaging system can facilitate detection of changes in depressed-type early gastric cancer. Gastrointest Endosc 2008;67:226-234.

10. Yoshizawa M, Osawa H, Yamamoto H, et al. Diagnosis of elevated-type early gastric cancers by the optimal band imaging system. Gastrointest Endosc 2009;69:19-28.

11. Demling L, Hagel HJ. Video endoscopy. Fundamentals and problems. Endoscopy 1985;17:167-169.

12. Yao K, Oishi T, Matsui T, Yao T, Iwashita A. Novel magnified endoscopic findings of microvascular architecture in intramucosal gastric cancer. Gastrointest Endosc 2002;56:279-284.

13. Yoshida S. Endoscopic diagnosis and treatment of early cancer in the alimentary tract. Digestion 1998;59:502-508.

14. Nakayoshi T, Tajiri H, Matsuda K, Kaise M, Ikegami M, Sasaki H. Magnifying endoscopy combined with narrow band imaging system for early gastric cancer: correlation of vascular pattern with histopathology (including video). Endoscopy 2004;36:1080-1084. 